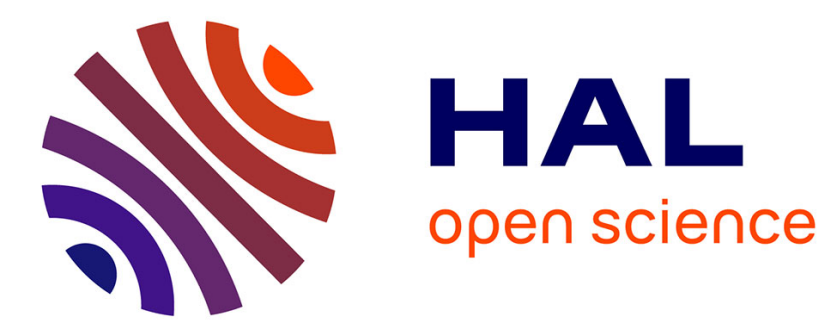

\title{
Le bois mort dans les paysages fluviaux français : éléments pour une gestion renouvelée
}

\author{
Yves-François Le Lay, Hervé Piégay
}

\section{To cite this version:}

Yves-François Le Lay, Hervé Piégay. Le bois mort dans les paysages fluviaux français: éléments pour une gestion renouvelée. Espace Géographique, 2007, 36 (1), pp.51-64. 10.3917/eg.361.0051. halshs-00366443

\section{HAL Id: halshs-00366443 \\ https://shs.hal.science/halshs-00366443}

Submitted on 6 Mar 2009

HAL is a multi-disciplinary open access archive for the deposit and dissemination of scientific research documents, whether they are published or not. The documents may come from teaching and research institutions in France or abroad, or from public or private research centers.
L'archive ouverte pluridisciplinaire HAL, est destinée au dépôt et à la diffusion de documents scientifiques de niveau recherche, publiés ou non, émanant des établissements d'enseignement et de recherche français ou étrangers, des laboratoires publics ou privés. 
Le Lay Y.-F. ${ }^{1,2}$ et Piégay H. ${ }^{2}$, 2007. "Le bois mort dans les paysages fluviaux français : éléments pour une gestion renouvelée". L’Espace géographique, 1, p. 51-64.

${ }^{1}$ Université de Lyon ; mél. : yf_lelay@ hotmail.com

${ }^{2}$ CNRS UMR 5600 «Environnement, Ville, Sociétés », 18 rue Chevreul, 69362 Lyon Cedex 07

\title{
Le bois mort dans les paysages fluviaux français : éléments pour une gestion renouvelée
}

Wood in French riverscapes: key elements for a new management perspective

\section{Résumé}

La montée de nouvelles préoccupations concernant le bois en rivière est analysée comme procédant de la négligence de l'entretien des cours d'eau français, ainsi que du reboisement spontané des marges fluviales. Les bois flottants sont une source de nuisances, mais leurs bienfaits écologiques sont aujourd'hui également démontrés. La législation et l'environnement socioculturel demeurent des freins à un renouveau de leur gestion. Cette étude montre que le bois mort reste négativement valorisé dans l'imaginaire collectif. Il importe d'en prendre conscience lorsque des décisions dites rationnelles doivent être prises en matière de gestion des rivières aménagées.

\begin{abstract}
This article focuses on the increasing interest in wood in rivers that follows the negligence of watercourse maintenance and the consequential spontaneous reforestation. In-channel wood may provoke damages, but its ecological benefits are also demonstrated. Within such a framework, preserving - indeed reintroducing wood, like in other geographical areas - seems difficult insofar as legislation and socio-cultural environment act as a brake on improving wood management in French watercourses. The article shows that dead wood is a negatively appreciated object in public perception. Such irrational factors may influence the decisionmaking about large wood removal.
\end{abstract}

Mots clés

Bois flottants, entretien des cours d'eau, évaluation des paysages de rivière, perception

\section{Keywords}

Woody debris, river channel maintenance, riverscape assessment, public perception

\section{Introduction}

Etant donné les conséquences néfastes qu'implique la suppression du bois mort lors du nettoyage des cours d'eau (Abe et Nakamura, 1999), sa réintroduction dans le cadre de programmes de restauration est devenue très populaire en Amérique du Nord (Etats-Unis et Canada), dans quelques pays européens (Allemagne, Autriche, Liechtenstein, Suède), en Australie et au Japon (Reich et al., 2003 ; Kail et Hering, 2005). Les objectifs sont alors d'améliorer les conditions d'habitat, de prévenir l'érosion de berge, de protéger les plantations d'arbres, de stocker le sédiment dans les torrents et d'imiter des «conditions naturelles ».

Le vocabulaire employé par les acteurs de la rivière pour y désigner le bois est particulièrement évocateur de leurs intérêts et de la représentation qu'ils ont du fonctionnement de l'hydrosystème. Dans la littérature scientifique, l'expression «large woody debris» a longtemps prévalu, jusqu'au premier colloque international sur le bois dans les rivières du monde en 2000, au cours duquel la communauté a reconnu que le terme de débris dépréciait un objet pourtant écologiquement bénéfique. L'expression «bois en rivière » tend ainsi aujourd'hui à être privilégié. En France, EDF sur le Rhin ou encore l'Agence de l'eau Adour-Garonne utilisent 
également l'expression « déchets flottants » (Lyaudet, 1995 ; Collectif, 2000) pour souligner le contenu varié des accumulations, tout en reconnaissant qu'ils sont constitués à 80-90\% de bois. Sur la Loire, Bachoc et al. (2002) préfèrent le terme d'«encombres », au sens d'«amas de matériaux (bois, obstacles divers, gros déchets...) ». Bien qu'ils se méfient de leur enlèvement systématique du fait de leur potentiel écologique, notamment en tant qu'habitat piscicole, les auteurs choisissent une dénomination qui souligne que le bois encombre le lit, réduit la capacité de l'écoulement des eaux et implique des risques hydrauliques (élévation de la ligne d'eau, création de zones de dépôt ou encore déstabilisation d'ouvrages). Plus fréquemment, les guides techniques emploient le terme d'«embâcle » (Boyer et al., 1998 ; Jund et al., 2000 ; Ledard et $a l ., 2001)$. Mais lui-même n'est pas indemne d'une dévalorisation. Au XVII ${ }^{\text {ème }}$ siècle, une bâcle se présente comme une barre de bois avec laquelle on ferme une porte ou une fenêtre par dedans, et un bâclage désigne la fermeture de l'accès d'un port ou du passage sur une rivière effectué au moyen de chaînes ou de câblages.

Bien que la première reconnaissance ministérielle de l'intérêt de l'embâcle de bois pour l'écosystème d'eau courante soit encore récente (Maridet et Collin-Huet, 1994), l'objectif de cet article est de rendre compte de la montée de nouvelles préoccupations concernant le bois en rivière, du fait de l'évolution des pratiques agro-pastorales, des changements paysagers et de l'intérêt écologique du bois flottant aujourd'hui bien identifié par les travaux scientifiques. Mais la législation et l'environnement socioculturel sont autant de freins à un renouveau de la gestion du bois dans les rivières françaises.

\section{Un renouveau des préoccupations liées au bois en rivière}

Depuis le milieu du XIX ${ }^{\text {ème }}$ siècle, le contexte socio-économique et l'occupation du sol dans les milieux ruraux européens ont connu d'importantes transformations au terme desquelles maintes parcelles riveraines de cours d'eau ont été marginalisées (Piégay, 1996).

\section{L'abandon de l'entretien des cours d'eau}

La rivière a longtemps fourni aux riverains des ressources inestimables, telles que l'eau, le sédiment ou le bois (par exemple Bravard, 1987 ; Valette et Gazelle, 2000). Ce dernier a été activement recherché au sein des communautés rurales. Selon sa qualité et ses dimensions, il avait plusieurs fonctions : bois de chauffe surtout, mais aussi bois de charpente à la faveur d'un bois flotté discrètement détourné, ou plus simplement matériau pour les outils agricoles, ceux de la cuisine et les meubles. Son ramassage faisait l'objet d'une réglementation précise. Par exemple, dans son titre XVII, la coutume du Nivernais (Coquille, 1610) laissait à la disposition des usagers le mort-bois qui « est tenu et réputé bois non portant fruit » et le bois mort qui « est bois chu, abattu, ou sec debout, qui ne peut servir qu'à brûler ». Une telle distinction montre suffisamment quelle valeur était conférée au bois. Vilacèque (2003) cite un document des archives de Sommières, dans le Gard : en 1336, le viguier accepte de se départir de son droit à exploiter le bois flotté. Les habitants peuvent «continuer à prendre le bois qui nagera sur les eaux pendant les inondations de Vidourle». Sur la lithographie de Barnard (figure 1), le tronc aide à rendre l'image divertissante grâce à un fait divers ; mais celle-ci souligne également qu'on prend des risques pour récupérer du bois jusque dans la rivière...

Le lit majeur des cours d'eau n'était pas abandonné. Bien au contraire, une activité pastorale occupait les terres inondables, la taille régulière des arbres contribuait à entretenir les berges et les embâcles étaient supprimés. Un recueil des lois en Languedoc (Albisson, 1781) permet d'en avoir une bonne idée. Le bois se déposant préférentiellement au droit d'obstacles, il s'accumulait facilement contre les chaussées. Selon une ordonnance du 7 février 1715, des plaintes dénoncèrent plusieurs particuliers qui venaient en prendre journellement auprès des chaussées du pont de Saint-Esprit, n'hésitant pas à s'attaquer aux protections elles-mêmes... 
« Nous [...] faisons défenses à toutes personnes de prendre aucune terre, sable, gravier \& bois, soit au devant ou au derrière desdits clayonnages, à peine de punition corporelle \& de tous dépens, dommages \& intérêts ». Et lorsque l'attrait de la ressource ne suffisait pas, un curage radical était porté à la charge de la province ou des communautés, dans le cadre de la lutte contre les débordements. Par exemple, face à l'encombrement du lit du Lauzon, de nombreux arrêts et ordonnances se succédèrent pour le désengorger et éviter l'endommagement du "grand chemin qui va de la ville du Saint-Esprit à la Palud ». Ainsi, le traité du 13 septembre 1714 préconisait d'ôter, couper et démolir les haies vives, les arbres, les pieux, les ouvrages « \& autres empêchements qui seront dans son lit », jusqu'au Rhône et dans toute sa largeur, pour donner un cours plus libre aux eaux. En 1744, un arrêt du Conseil d'Etat du Roi interdit toute plantation et tout ouvrage qui pussent freiner les eaux, que ce fût dans le lit, sur les bords du ruisseau ou dans les bras morts.

Cependant, depuis un demi-siècle, les rivières sont de moins en moins entretenues. Le propriétaire est connecté aux différents réseaux d'adduction d'eau potable, d'assainissement, d'évacuation des déchets ou encore d'énergie. Le cours d'eau, hier ressource pour le propriétaire du lit, devient souvent une contrainte. Le riverain n'a plus intérêt à l'entretenir, et bien souvent ne l'entretient plus malgré la législation actuelle. Désormais exclu du secteur de l'énergie et de celui des matériaux, le bois mort n'est plus branché sur les circuits d'échanges productifs, mais appartient à la catégorie peu flatteuse des «inutiles au monde » qui y séjournent sans vraiment y appartenir.

\section{Les dynamiques spatiales des ripisylves}

Depuis 1850, le couvert végétal des parcelles riveraines a connu une dynamique progressive liée à la déprise agraire. Les labours y ont cédé la place à des zones pâturées qui se sont progressivement enfrichées ; et des landes boisées ont finalement été supplantées par les taillis (Piégay, 1996). A la production exigeante des céréales s'est substitué un espace en déshérence qui sert au mieux de parcours pour les bêtes. Particulièrement après 1945, la motorisation et la simplification des productions agricoles ont contribué à ce recul sur les terres inondables et à la fermeture du paysage par boisement spontané.

Si une reconquête forestière est à l'œuvre au bord de nombreux cours d'eau européens (Piégay et al., 2003), cette évolution varie d'un contexte géographique à l'autre. Elle n'a pas été observée sur les cours d'eau dont les marges restent valorisées à des fins agricoles ou pastorales (par exemple sur le Doubs) ou ceux sur lesquels les crues ont été contrôlées de telle sorte qu'une implantation humaine s'est développée sur la rive immédiate du lit mineur (comme l'Isère et le Rhône). Mais, globalement, la forêt alluviale s'étend et tend à vieillir : elle atteint fréquemment 50 ans (figure 2). Ces mutations favorisent les entrées croissantes de bois dans les hydrosystèmes et, sur la Durance par exemple, poussent à reconsidérer la gestion des boisements riverains (Boyer, 2005).

\section{Un arsenal législatif pour entretenir la rivière}

L'entretien des cours d'eau est une action courante et bénéficie d'un cadre juridique élaboré progressivement. Le texte-clef est l'avis du Conseil d'Etat du 27-30 pluviôse an XIII (19 février 1805). Etant donné que les propriétaires riverains «sont exposés à tous les inconvénients attachés au voisinage des rivières non navigables », il leur donna le droit de pêche et rappela leur assujettissement à la dépense du curage et à l'entretien de ces rivières. Cet avis reconnaissait le vieil adage «ubi onus, ibi emolumentum », qui supporte les charges doit avoir les bénéfices.

De même, la loi du 29 juin 1984 relative à la pêche en eau douce et à la gestion des ressources piscicoles repose sur les principes de l'équité naturelle, puisque «tout propriétaire d'un droit de pêche, ou son ayant cause, [...] doit effectuer les travaux d'entretien, sur les berges et dans le lit du cours d'eau, nécessaires au maintien de la vie aquatique ». Mais ce n'est qu'avec 
la loi Barnier, du 2 février 1995 et relative au renforcement de la protection de l'environnement, que le bois devient véritablement un objet juridique. Le «propriétaire est tenu à un curage régulier pour rétablir le cours d'eau dans sa largeur et sa profondeur naturelles, à l'entretien de la rive par élagage et recépage de la végétation arborée et à l'enlèvement des embâcles et débris, flottants ou non, afin de maintenir l'écoulement naturel des eaux, d'assurer la bonne tenue des berges et de préserver la faune et la flore dans le respect du bon fonctionnement des écosystèmes aquatiques ».

La législation française relative à l'entretien est singulière en ceci qu'elle continue à justifier ces interventions, non seulement en termes de sécurité publique mais aussi de préservation écologique, ce qui est désormais largement infirmé par la communauté scientifique.

\section{Nuisances, contraintes et risques liés au bois en rivière}

Derrière la législation des cours d'eau, il y a une vision organiciste, fréquente aux $\mathrm{XVIII}^{\text {ème }}$ et $\mathrm{XIX}^{\text {ème }}$ siècles, selon laquelle la nature a créé les ruisseaux et les rivières pour vivifier le globe. La devise en est de laisser couler l'eau. Le cours des eaux est, dans le grand corps de la terre, ce qu'est la circulation du sang dans le corps humain. «Si vous arrêter les fluides, vous détruisez dans l'homme le principe de la vie, et sur la terre vous causez des engorgements, vous faîtes extravaser les eaux, et vous créez des marais pestilentiels, fléaux de l'agriculture et de l'humanité » (Heurtault-Lamerville, 1790).

La réglementation mise en ouvre sur le Lauzon au XVIII ${ }^{\text {ème }}$ siècle et présentée précédemment n'a rien d'exceptionnel; elle permet néanmoins de relever tout à la fois la difficulté et la nécessité de libérer le cours des eaux. Avant les années 1970, le bois en rivière était largement considéré comme une nuisance à cause de ses effets hydrauliques et des risques associés. Le bois était systématiquement supprimé des chenaux afin de satisfaire les différents usages qui s'y pratiquaient. Par exemple, des tronçons de rivière étaient régulièrement nettoyés sur un grand linéaire pour prévenir les embâcles et favoriser ainsi la navigation et le flottage de bois (Benoit et al., 2004). Par ailleurs, le riverain tirait un bénéfice de cette ressource et la tâche que lui avait assignée le législateur ne constituait pas véritablement une contrainte. L'opération s'avérait utile pour maintenir la stabilité des berges en favorisant les systèmes racinaires aux dépens des systèmes aériens et pour pérenniser les usages pratiqués sur le milieu riverain. Ces pratiques d'entretien n'étaient bien sûr pas l'apanage des propriétaires et gestionnaires français, comme le révèlent le Code municipal du Québec (art. 783), la loi wallonne relative aux cours d'eau non navigables (en date du 28 décembre 1967) et la loi du canton de Fribourg sur l'aménagement des eaux (en date du 26 novembre 1975). L'objectif de l'entretien est aujourd'hui également de protéger les infrastructures de franchissement de divers dommages (Diehl, 1997).

\section{Le bénéfice écologique du bois en rivière}

Au cours des trois dernières décennies, le bois en rivière a fait l'objet de nombreuses études scientifiques qui ont mis en lumière ses atouts écologiques (Gregory et al., 2003), particulièrement dans les hydrosystèmes tempérés. L'intérêt du bois est de complexifier la morphologie du cours d'eau, formant ainsi des habitats faunistiques intéressants, tout en retenant la matière organique en transit : il affecte positivement l'ensemble de la chaîne trophique.

\section{Diversification des formes de la rivière}

L'entrée de bois dans le chenal des cours d'eau se fait à la faveur de sapements de berge, de coups de vent et de chutes de branches. Des phénomènes paroxystiques, tels que des laves torrentielles, des inondations et des feux de forêt sont susceptibles d'introduire de grandes quantités de bois dans le chenal pendant de courtes périodes (Bilby et Bisson, 1998). La taille du cours d'eau joue sur la localisation, les caractéristiques et les fonctions des bois flottants. Dans 
les cours d'eau pentus des têtes de bassin, les troncs obstruent toute la largeur du chenal et créent un profil en long en escalier qui contrôle le stockage et la libération des sédiments et des détritus. Quand le chenal devient trop large pour être obstrué par de grands troncs, les accumulations de bois se forment sur ses marges. La dégradation des fûts s'effectue alors dans le chenal de différentes façons : décomposition microbienne, fragmentation par des invertébrés, abrasion physique et transport vers l'aval.

Les embâcles se localisent généralement dans le lit mineur, en berge ou dans le chenal. S'accumulant à la faveur d'un obstacle, l'embâcle renforce celui-ci et détermine le champ de vitesses au niveau de la section (figure 3). Il provoque un ralentissement des écoulements et une élévation de la ligne d'eau à l'amont. Si le chenal n'est pas entièrement obstrué, l'embâcle concentre l'écoulement, ce qui suscite une accélération de ce dernier au droit de l'obstacle. En cas de surverse, l'accumulation de bois se comporte comme un déversoir. Si une fosse de dissipation de l'énergie existe à l'aval, une recirculation et des tourbillons peuvent être observés (Maridet $e t$ al., 1996).

Les grandes pièces de bois contrôlent ainsi la géométrie du chenal, notamment la largeur et la profondeur. Dans les petits cours d'eau, elles contribuent à créer des mouilles et des chutes d'eau (Bilby et Bisson, 1998). Les relations entre les bois flottants, la superficie des mouilles et la distance qui les sépare varient avec la pente et la largeur du chenal (Beechie et Sibley, 1997). Bien que les bois flottants soient le principal agent de formation des mouilles, leur rôle hydraulique dépend de leur taille : les pièces volumineuses et immobiles contribuent davantage à la formation des mouilles que les petits débris mobiles (Urabe et Nakano, 1998). Si l'abondance de bois flottants augmente, l'espacement entre les mouilles diminue et la surface qu'elles occupent augmente, surtout lorsque la pente du chenal est modérée. Les chenaux à faible pente sont moins sensibles à l'abondance de bois flottants parce que leurs mouilles se forment selon d'autres mécanismes si les bois flottants manquent. D'autre part, la taille des bois qui ont formé les mouilles augmente avec la largeur du chenal, mais n'est pas liée à la pente.

Sur les grands cours d'eau, le bois peut aussi affecter localement la forme du chenal. Les embâcles accumulés à l'apex des bancs stables et au niveau des méandres modifient localement l'hydraulique de l'écoulement, et donc les caractéristiques spatiales des phénomènes d'érosion et de dépôt qui conduisent à la formation de mouilles et de bancs. Par exemple, un embâcle constitué sur l'apex d'un banc est associé à une mouille d'érosion en forme de croissant, un banc à l'amont et un banc central à l'aval où se développe la ripisylve (Abbe et Montgomery, 1996).

\section{Le bois comme structures de rétention}

Le bois en rivière fonctionne comme une structure de rétention face aux sédiments, aux matériaux organiques tels que les feuilles ou les brindilles, ou tout autre corps flottant (figure 3). L'embâcle modifie localement la géométrie, diversifie ainsi les processus de transport solide et augmente l'hétérogénéité de la granulométrie du lit. A l'amont, il piège et stocke non seulement la charge de fond, mais encore les particules fines, les limons et les sables : la granulométrie est donc généralement de plus petite taille. Au droit de l'embâcle, les forces tractrices augmentent et les fines sont prises en charge: la granulométrie y est alors plus grossière. De même, les particules grossières sont plus apparentes à l'aval immédiat de l'obstacle.

Près de $40 \%$ des pièces de bois sont associés à des accumulations sédimentaires dans les chenaux larges de moins de $7 \mathrm{~m}$, moins de $30 \%$ dans les chenaux larges de 7 à $10 \mathrm{~m}$ et moins de $20 \%$ dans les chenaux dont la largeur dépasse $10 \mathrm{~m}$ (Bilby et Ward, 1989). La surface des accumulations sédimentaires et le volume de la pièce de bois suscitant le dépôt sont liés quelle que soit la taille du cours d'eau. Mais la relation est plus claire dans les grands chenaux; et la quantité de sédiments et de matière organique fine retenue par les bois flottants décroît avec l'augmentation de la taille des cours d'eau. En outre, la suppression de barrages de bois peut provoquer une vive remobilisation des sédiments. Dans le cas d'un cours d'eau de l'Oregon, la 
turbidité et la quantité de matière en suspension ont augmenté pendant les crues d'hiver qui ont suivi l'enlèvement des bois; plus de $5000 \mathrm{~m}^{3}$ de sédiment ont ainsi été érodés sur un tronçon de $250 \mathrm{~m}$ (Beschta, 1979).

La présence de fûts dans le chenal favorise d'autre part la rétention de la matière organique particulaire, soit directement en constituant des barrages de bois, soit indirectement par la modification de la morphologie du chenal et la création de zones de dépôt (Bilby et Bisson, 1998). Bilby et Likens (1980) ont ainsi montré combien les barrages de bois sont un élément important de l'écosystème des petits cours d'eau : ils piègent $75 \%$ de la matière organique dans les cours d'eau de rang 1, 58\% dans ceux de rang 2 et $20 \%$ dans ceux de rang 3 . Les accumulations de matière organique particulaire associées aux accumulations de bois sont plus fréquentes dans les petits cours d'eau, mais plus volumineuses dans les grands cours d'eau.

Etant donné que la majorité des entrées d'énergie dans un cours d'eau hétérotrophe se produit en automne lors de la chute des feuilles, la capacité de rétention liée aux structures ligneuses s'avère primordiale en fournissant des réservoirs de nourriture et une source de carbone disponible toute l'année. Le temps de résidence des matières organiques liées au piégeage des bois est plus long que celui lié aux autres substrats non minéraux. En retenant cette matière organique, ils lui permettent de s'affiner dans les têtes de bassin plutôt que d'être transportée sous forme de particules grossières.

\section{L'influence du bois sur les communautés d'insectes}

Les entrées allochtones, et particulièrement les feuilles, sont une source d'énergie importante pour les communautés de macro-invertébrés. Dans les hydrosystèmes tempérés et forestiers, celles-ci sont dépendantes de la dégradation de la matière organique grossière. Une relation positive liant la biomasse de nombreux insectes et la quantité de détritus benthiques (Minshall et Minshall, 1977) a été décrite dans le cadre du concept de continuum fluvial (Vannote et al., 1980). Plusieurs groupes alimentaires fonctionnels évoluent en bénéficiant de la dégradation des plus grosses particules par les détritivores.

La présence de bois dans les chenaux influe sur la biomasse de macro-invertébrés benthiques et sur la composition spécifique de leur communauté, parce que les fûts stables sont pour eux un habitat important, dans les cours d'eau tant à charge fine qu'à charge grossière. Angermeier et Karr (1984) ont constaté que les invertébrés benthiques sont significativement plus abondants dans une zone où des bois flottants ont été ajoutés que dans celle où ils ont préalablement été supprimés. Les bois flottants sont ainsi colonisés par de nombreux invertébrés, notamment des chiromides, des trichoptères et des éphéméroptères.

L'influence du bois peut être précisée en fonction de sa nature. Les bois immergés fournissent des points d'attache stables et des refuges (Borchardt, 1993) dans les cours d'eau à charge fine et à substrat instable, et plus généralement fonctionnent comme une source de nourriture pour les xylophages. Ils fournissent également un substrat pour la production de biofilm (Golladay et Sinsabaugh, 1991). Et les bois flottants servent de refuge aux organismes emportés lors des forts débits, lorsque les substrats immergés sont sujets à l'érosion. Sur une rivière traversant un environnement désertique, Haden et al. (1999) ont aussi montré qu'ils constituent un important substrat pour les macro-invertébrés. Si la biomasse de ces derniers n'est pas significativement différente entre les bois flottants et le fond d'un lit graveleux, il existe des différences dans l'assemblage des éphéméroptères qui sont attribuées au type de ressources alimentaires disponibles au sein de chaque habitat : les bois sont dominés par des invertébrés râcleurs et les galets par des filtreurs. Ainsi, les bois flottants diversifient les types de niches disponibles pour les macro-invertébrés dans les systèmes lotiques turbides.

Les arbres tombés modifient également la géomorphologie des cours d'eau et créent dans leur environnement proche une diversité d'habitats pour de nombreux taxons. Etant donné que la présence de bois flottants joue sur l'habitat et les relations trophiques en augmentant la 
proportion de mouilles, elle influe également sur la structure des communautés de groupes alimentaires fonctionnels : les collecteurs dominent dans les mouilles et les déchiqueteurs dans les radiers. En effet, la rétention des matériaux organiques favorise une dégradation plus complète des feuilles par les déchiqueteurs. Des particules organiques plus fines sont alors disponibles pour d'autres groupes alimentaires fonctionnels. Dans ce sens, Lemly et Hilderbrand (2000) ont observé que les déchiqueteurs sont mieux représentés dans les radiers, qui possèdent des fragments de matière organique en quantité moindre mais de taille plus grossière que les mouilles. Les détritus entretiennent les relations suivantes avec la matière organique particulaire: positive avec les collecteurs, nulle avec les prédateurs et négative avec les déchiqueteurs et les filtreurs.

\section{Le bois comme habitat piscicole}

Le bois fournit de la nourriture, des refuges en période de hautes eaux, une couverture protectrice contre les prédateurs, et plus généralement il augmente la disponibilité et la diversité des structures d'habitat aquatique aux différents stades des cycles de vie des poissons (figure 3). Les mouilles formées par des structures ligneuses fournissent dans les cours d'eau un habitat-clé pour de nombreuses espèces de poissons, mais le bois mort peut aussi être utilisé comme abri (Bilby et Bisson, 1998).

Les bois flottants diversifient la structure spatiale des mosaïques d'habitats fonctionnels dont l'utilisation nycthémérale est fonction du type d'activité du poisson (repos, alimentation et protection contre les prédateurs, compétition inter-spécifique). Ces structures affectent ainsi la répartition, la croissance, la reproduction et la survie des truites communes (Salmo trutta L). Les bois flottants favorisent la formation de mouilles, qui sont considérées comme le type d'habitat préféré des salmonidés. Urabe et Nakano (1998) ont ainsi montré que la biomasse de truites arcen-ciel est corrélée au volume des mouilles, lui-même étant positivement corrélé au volume des bois flottants.

L'influence du bois varie également en fonction de la taille du cours d'eau. Dans un petit cours d'eau de l'Illinois, Angermeier et Karr (1984) ont montré que les plus gros poissons évitent les tronçons sans bois. Cette relation positive entre poissons et bois flottants semble être plus fermement liée aux avantages que le bois procure en termes de caches qu'à une disponibilité accrue de nourriture ou à une protection plus efficace vis-à-vis de conditions hydrauliques extrêmes. D'autre part, au terme d'une étude sur la reproduction de la truite dans un torrent à forte pente qui présente peu de zones de frayères, Champigneulle et al. (2003) estiment que les embâcles sont favorables à une diversification spatio-temporelle des sites de frai : ils pourraient être une composante essentielle à la survie des populations de truites en milieu torrentiel dans une large gamme de conditions extrêmes, comme les crues, sécheresses, érosions...

Dans les grands cours d'eau, les habitats piscicoles sont également bien associés aux accumulations de bois le long des berges et dans le système de chenaux secondaires de la plaine d'inondation. Les poissons sont aussi présents derrière et sous les très grandes pièces de bois qui se déposent contre les bancs de graviers du chenal principal et forment des mouilles associées (Bisson et al., 1987). Les ambiances comportant du bois abritent ainsi un peuplement plus diversifié et des individus plus nombreux (figure 4). Le vairon, le chevesne, le spirlin ou encore la perche commune présentent une «attirance » pour le bois en rivière (Thévenet, 1998).

Les travaux de nettoyage du lit et des abords du cours d'eau ont un effet négatif sur les peuplements en réduisant le nombre d'abris indispensables au maintien des poissons. Ainsi, la suppression de la ripisylve, combinée avec le nettoyage complet du cours d'eau et un bûcheronnage à courte rotation, a pour conséquence de modifier les sources, les mécanismes d'alimentation et la redistribution des bois dans l'hydrosystème; ce qui conduit à altérer la biomasse et la biodiversité spécifique des poissons (Bisson et al., 1987). Sur les secteurs étudiés 
par ces auteurs, la chute d'arbres, en reconstituant des caches, a permis de revenir à la densité initiale avant nettoyage.

\section{La perception négative du bois mort dans les paysages fluviaux}

Bien que le bois constitue un bienfait écologique, les services de l'Etat continuent à promouvoir l'entretien des cours d'eau et maintiennent un cadre juridique aujourd'hui désuet. Une approche de géographie dite humaniste, basée sur les productions artistiques, a ainsi été conduite afin de mieux comprendre le contexte culturel dans lequel s'inscrivent les pratiques d'entretien. Les résultats soulignent l'influence négative du bois mort sur la perception des paysages fluviaux dans le cadre de la culture dite profane.

\section{De l'arbre au bois flottant : une scénographie perceptive}

La place du bois mort et des corps flottants dans l'imaginaire collectif de l'arbre et de la rivière montre combien un objet a priori anodin peut être révélateur d'un anthropocentrisme susceptible d'influencer les prises de décision et les pratiques liées à la gestion des cours d'eau.

C'est au terme d'une dégradation, dont la scénographie perceptive peut être retracée, que l'arbre est réduit à l'état de débris; et les hommes n'y sont pas insensibles. Spontanément, une analogie anatomique, physiologique et psycho-affective associe les hommes et les arbres (Eliade, 1964 ; Dumas, 2002). L'arbre est humanisé, et n'est jamais repoussant : «Raisons pour lesquelles un bourgeon, pourtant poisseux à ses débuts, ne dégoûte pas quand on le touche [...]. Raisons pour lesquelles il est rare de dire d'un arbre qu'il est sale »(Quintane, 1999). Si l'arbre peut afficher sa souffrance dans un port tourmenté, il reste immortel.

Quand il meurt, malgré tout, il rappelle à l'humanité l'absurdité et la fragilité de sa condition. "Vieux peuplier, / Tu es tombé / Dans le miroir / De l'eau dormante. / Je t'ai vu sombrer / Dans le crépuscule / Et j'écris pour toi cette plainte / Qui est aussi la mienne » (Peuplier mort, 1920, dans Garcia Lorca, 1987). Le registre ontologique du bois mort est clair : considérer cet objet, c'est voir ce qui nous perd et dont il faut s'éloigner. «Raisons pour lesquelles dessiner un arbre mort = difficultés psychologiques » (Quintane, 1999). Le bois mort inspire du dégoût, de la répulsion et suscite un souhait de séparation et d'éloignement.

La civilisation occidentale et son hygiénisme conduisent à échapper au délétère, aux matières salissantes et informes. "Nos sociétés amplifient ce puritanisme matériel, comme s'il fallait partout nettoyer, éliminer et même brûler » (Dagognet, 1997). Dans ce contexte, apprécier le bois mort, ce serait célébrer le putride, l'émietté, le déchiqueté, le fragmentaire et le brisé. En effet, le bois mort est le résultat de la désorganisation d'une unité bien organisée, ce qui suscite l'émotion de l'homme face à la décomposition et la fétidité : «La malignité des choses informes est un mal trop grand pour être dit » (Yeats, 1899).

Du fait de ses relations à l'arbre et de la charge sensible inhérente à l'eau, le bois mort est perçu très différemment en rivière et en forêt. La précellence de l'eau en fait l'élément pur; elle est féminine et maternelle (Bachelard, 1942 ; Jung, 1953 ; Durand, 1992). Or, le bois mort noircit l'ensemble éthéré de la rivière. Laissé à lui-même, misérable et fangeux, il glisse vers la noirceur et sert de signe avant-coureur à la décomposition cadavérique. Cette dimension putride n'a pas échappé aux artistes, notamment à J. Bosch dont l'homme-arbre du Jardin des délices est un élément fort du volet de l'enfer et du troisième acte de la chute... Ses jambes transformées en souches d'arbres morts sont plantées dans des barques comme dans des sabots. Et son torse est fait d'une écorce vide en forme de coquille d'œuf, ce qui signifie le manque de valeur et représente le péché.

\section{Le bois en rivière, infra-objet de l'imaginaire collectif}


Le bois en rivière est d'abord un bois mort, et les actions sur l'eau courante s'ancrent dans l'angoisse du temps qui passe et de la fin irrémédiable. Selon Bachelard (1942), « [...] le fétu emporté par le ruisseau est l'éternel symbole de l'insignifiance de notre destin». Trois complexes, empruntés à cet auteur, font du bois en rivière des images de la mort acceptée, de la mort désirée et de la mort provoquée.

Le bois à la dérive réveille chez les poètes le motif de la mort acceptée qui s'ancre dans le complexe de Charon. Le voyage sur le fleuve alimente le mythe universel du dernier voyage ; et l'arbre emporté par le courant actualise ce motif... Déjà marqué par «l'isolement de l'arbre au bord de l'eau » (Clerbout, 1996), l'observateur ne peut qu'être ému par sa chute et plus encore par sa nouvelle mobilité sur l'eau : «L'arbre / déraciné / pour un départ 》 (L'arbre mémoire, dans Bessière, 1979). La référence aux travaux de X. de Saintine a souvent été faite pour décrire les pratiques (perçues comme diverses et étranges) dont les Celtes usaient vis-à-vis des dépouilles humaines afin de les faire disparaître. Vers 1560, des ouvriers hollandais, en fouillant un atterrissement du Zuiderzee, découvrirent plusieurs troncs d'arbres conservés par pétrification. Chacun de ces troncs avait conservé quelques débris d'un homme, eux-mêmes presque fossilisés. «Evidemment, c'était le Rhin, ce Gange de l'Allemagne, qui les avait charriés jusque-là, l'un portant l'autre » (de Saintine, cité dans Bachelard, 1942). Cette pratique mêle un culte de l'arbre, qui permet de passer de la terre vers le ciel, et un culte de la rivière, en faisant don d'un corps à l'eau. D'une certaine manière, en plaçant le mort au creux de l'arbre, puis en confiant l'arbre aux eaux, les puissances maternelles sont doublées.

Un simple bois flottant évoque également chez le rêveur la figure du noyé et nourrit le complexe d'Ophélie, en référence à la pièce écrite par Shakespeare en 1601. Le bois absorbé par l'eau appartient au système des images de la mort désirée : «Ophélie, Ophélie / Ton beau corps sur l'étang / C'est des bâtons flottants / A ma vieille folie » (Laforgue, 1887). Plus précisément, l'engloutissement du bois mort dans une eau elle-même morte évoque le suicide et la dissolution totale. Ophélie et les bois flottants sont assimilés par une anthropomorphisation spontanée. Les motifs de l'enlacement et de l'engloutissement procèdent d'observation quasi-quotidienne de l'eau absorbant et enfantant le soleil. Selon Anaximandre, les choses retournent à ce dont elles sont sorties : « Je suis le maître / D'un bateau / Qui n'est pas mien / Et qui s'enfonce / Corps et biens » (Seconde Traversée, dans Balinec, 1991). C'est dans l'eau que s'achève la boucle. Nés de l'argile, c'est-à-dire d'un mélange de terre et d'eau, les hommes retournent à l'argile, avant de revenir à l'eau des commencements.

Enfin, conformément au complexe de Swinburne, la rivière est une école de l'effort où la mort peut être provoquée et dépassée. Le bois y matérialise le monstre, le cache, mais aussi trahit sa présence. Le corps flottant est inséparable du danger. Il révèle ce que nous ne voulons pas reconnaître en nous, cette part insupportable qui nous constitue. La surface sur laquelle il repose est une entrée vers les enfers, vers l'antre du monstre. S'il s'agit d'une épreuve, celle-ci peut être surmontée... Par exemple, en aval d'un pont qui enjambe la Midouze à Mont-de-Marsan, Hirakawa a conçu un ample projet d'art environnemental (figure 5) pour un site dégradé en dessinant une succession de cinq ellipses composées soit de la terre rouge des arènes locales, soit d'entassements alternés de souches de chêne et de billes de pins maritimes. Par cette œuvre qui représentait un monde imparfait parsemé de vides, l'artiste a fait le deuil de son père décédé quelques mois plus tôt. Le terme «ellipse » désigne l'omission d'un ou plusieurs mots en les sous-entendant, la forme dit l'absence et le manque ; et le nombre, comme les cinq sens, évoque un repli de l'être sur la dialectique essentielle entre la vie et la mort. L'organicité des moignons d'arbre doit réveiller un espace qui se substitue ainsi à l'inexistence. S. Hirakawa dresse une nouvelle généalogie : l'arbre y révèle la prise de conscience du monde tel qu'il est, notamment de l'inexistence, de la désintégration et des manques.

\section{Conclusion}


Les études concernant le bois en rivière (figure 6) sont abordées dans un cadre systémique, à l'interface du système fluvial (Schumm, 1977), ou plus largement de l'hydrosystème (Roux, 1982 ; Amoros et Petts, 1993), et du sylvo-système (Houzard, 1985). Les connexions longitudinale et latérale se manifestent non seulement par les sapements de berge et l'inondation des forêts alluviales, mais aussi par les entrées de bois dans le chenal. Cependant, cette approche qui identifie les facteurs régissant ces phénomènes, gagne à reconnaître toute l'importance des représentations et pratiques humaines telles qu'elles sont considérées dans le cadre des sociosystèmes (Lapierre, 1992). Plus globalement, travailler sur les interactions entre les communautés riveraines et les milieux d'eau courante contribue à donner tout son sens au concept englobant d'anthroposystème (Lévêque et al., 2003) et ouvrir plus largement le débat sur la gestion du bois en rivière.

\section{Références bibliographiques}

ABbe T. B., Montgomery D. R. (1996). «Large woody debris jams, channel hydraulics and habitat formation in large rivers ». Regulated Rivers, 12, p. 201-221.

ABE T., NAKAMURA F. (1999). «Effects of experimental removal of woody debris on channel morphology and fish habitat ». Ecology and Civil Engineering, 2, p. 179-190.

ALBISSON J. (1781). Lois municipales et économiques de Languedoc. Montpellier : Rigaud et Pons, 2 tomes, 674 et $702 \mathrm{p}$.

Amoros C., PetTs G.E. (1993). Hydrosystèmes fluviaux. Paris : Masson, 300 p.

ANGERMEIER P. L., KARR J. R. (1984). «Relationship between woody debris and fish habitat in a small warmwater stream ». Transactions of the American Fisheries Society, 113, p. 716-726.

BACHELARD G. (1942). L'eau et les rêves. Essai sur l'imagination de la matière. Paris : José Corti, $221 \mathrm{p}$.

Bachoc A., Guillou S., Maman L., Bacchi M., Berton J.-P., Fauconnier J.-M. (2002). Restauration et entretien du lit de la Loire et de ses affluents. Guide Méthodologique. Plan Loire Grandeur Nature, DIREN Centre, Service de Bassin Loire-Bretagne, 2 vol., 68 et 105 p.

BALINEC Y. (1991). Ponderale. Plouzané : Editions An Amzer, n.p.

BeECHIE T. J., SiBLEY T. H. (1997). «Relationships between channel characteristics, woody debris, and habitat in northwestern Washington streams ». Transactions of the American Fisheries Society, 126, p. 217-229.

Benoit P., Berthier K., BoËt P., Rezé C. (2004). «Les aménagements hydrauliques liés au flottage du bois, leur impact sur le milieu fluvial (XVI ${ }^{\mathrm{e}}-\mathrm{XVIII}{ }^{\mathrm{e}}$ siècles) », dans Fleuves et marais, une histoire au croisement de la nature et de la culture. Sociétés préindustrielles et milieux fluviaux, lacustres et palustres : pratiques sociales et hydrosystèmes, Burnouf J., Leveau P. (dir.). Paris : C.T.H.S., p. 311-320.

BeschtA R. L. (1979). «Debris removal and its effects on sedimentation in an Oregon Coast Range stream ». Northwest Science, 53, p. 71-77.

BESSIERE J. (1979). L'arbre mémoire. Eygalières : Editions du Temps Parallèle, 85 p.

BILBY R. E., BISSON P. A. (1998). «Function and distribution of large woody debris », dans River ecology and management: lessons from the Pacific coastal ecoregion, NAIMAN R. J., BILBY R. E. (dir.). New York : Springer-Verlag, p. 324-347.

BILBY R. E., LIKENS G. E. (1980). «Importance of organic debris dams in the structure and function of stream ecosystems ». Ecology, 61, p. 1107-1113.

BILBY R. E., WARD J. W. (1989). «Changes in characteristics and function of woody debris with increasing size of streams in western Washington ». Transactions of the American Fisheries Society, 118, p. 368-378.

Bisson P. A., Bilby R. E., Bryant M. D., Dolloff C. A., Grette G. B., House R. A., MurPhy M. L., KosKi K. V., SEdell J. R. (1987). «Large woody debris in forested streams in the Pacific Northwest: past, present, and future », dans Streamside Management: Forestry and 
Fishery Interactions, Salo E. O., Cundy T. W (dir.). Seattle et Washington : University of Washington, Institutes of Forest Ressources, p. 143-190.

BORCHARDT D. (1993). «Effects of flow and refugia on drift loss of benthic macoinvertebrates : implications for habitat restoration in lowland streams ». Freshwater Biology, 29, p. 221-227.

BOYER M., 2005. Etudes et expérimentations de nouvelles modalités d'essartement. Rapport d'étude, Contrat de rivière du Val de Durance, Syndicat Mixte d'Aménagement de la Vallée de la Durance, $140 \mathrm{p}$.

Boyer M., Piegay H., Ruffinoni C., Citterio A., Bourgery C., Caillebote P. (1998). Guide Technique SDAGE - La Gestion des Boisements de Rivière : Dynamique et Fonctions de la Ripisylve. Lyon : Agence de l'Eau RMC, fascicule I, 42 p.

BRAVARD J.-P. (1987). Le Rhône du Léman à Lyon. Lyon : La Manufacture, 452 p.

Champigneulle A., Largiader C. R., CAudron A. (2003). «Reproduction de la truite (Salmo trutta L.) dans le torrent de Chevenne, Haute-Savoie. Un fonctionnement original ?». Bulletin Français de la Pêche et de la Pisciculture, 369, p. 41-70.

Clerbout M. (1996). Pensé au bord de l'eau. Mortemart : Rougerie, 85 p.

COLLECTIF (2000). «Les déchets flottants ». Aquadour. Bulletin d'information sur l'eau dans le Bassin de l'Adour, 24, 4 p.

CoQuille G. (1610). Coutumes du Pays et Duché de Nivernais, http://membres.lycos.fr/brunodevoucoux/coutumeNivernais.htm.

Dagognet F. (1997). Des détritus, des déchets, de l'abject. Une philosophie écologique. Paris : Institut Synthélabo, 201 p.

DIEHL T. H. (1997). Potential drift accumulations at bridges. Washington : U.S. Federal Highway Administration Publication, $\mathrm{n}^{\circ}$ FHWA-RD-97-028, $114 \mathrm{p}$.

DumAs R. (2002). Traité de l'arbre. Essai d'une philosophie occidentale. Arles : Actes Sud, $255 \mathrm{p}$.

DURAND G. (1992). Les structures anthropologiques de l'imaginaire. Paris : Dunod, 536 p.

EliAdE M. (1964). Traité d'histoire des religions. Paris : Payot, 393 p.

GARCIA LORCA F. (1987). Oeuvres complètes. Paris : Gallimard, tome 1, 1894 p.

Golladay S. W., SinSABaugh R. L. (1991). «Biofilm development on leaf and wood surfaces in a boreal river ». Freshwater Biology, 25, p. 437-450.

Gregory S. V., BoYer K., Gurnell A. M. (dir.) (2003). The Ecology and Management of Wood in World Rivers. Bethesda : American Fisheries Society, 431 p.

Haden G. A., Blinn D. W., Shannon J. P., Wilson K. P. (1999). « Driftwood: an alternative habitat for macroinvertebrates in a large desert river ». Hydrobiologia, 397, p. 179-186.

Heurtault-LAmerville M. (1790). « Premier rapport sur le code rural, fait au nom du Comité d'agriculture et de commerce, le 29 août 1790 ». Archives parlementaires, p. 410-413.

HouZARD G. (1985). «Sylvosystème et sylvofaciès : essai d'étude global du milieu forestier », dans Phytosociologie et Forêsterie, RAMEAU J.-C., GeHU J.-M. (dir.). Nancy: Colloques phytosociologiques XIV, p. 231-236.

Jund S., Paillard C., Frossard P.-A., Lachat B., Saucy M., Jost G. (2000). Guide de gestion de la végétation des bords de cours d'eau. Rapport général. Moulins-Lès-Metz : Agence de l'eau Rhin-Meuse, $54 \mathrm{p}$.

JUNG C. G. (1953). Métamorphoses de l'âme et ses symbols. Genève : Georg, 770 p.

KAIL J., HERING D. (2005). «Using large wood to restore streams in Central Europe: potential use and likely effects ». Landscape Ecology, 20, p. 755-772.

LAFORGUe J. (1887). Moralités légendaires. Paris : Mercure de France, édition de 1924, 319 p.

LAPIERRE J.-W. (1992). L'analyse des systèmes. L'application aux sciences sociales. Paris : Syros, $229 \mathrm{p}$.

Ledard M., Gross F., Haury J., Lafontaine L., Hubaud M.-O., Vigneron T., Dubos C., Aubry M., Labat J.-J., Nioche-Seigneuret F., Vienne L., Craipeau F. (2001). Restauration 
et entretien des cours d'eau en Bretagne. Guide technique. Rennes : DIREN Bretagne et Bègles, Société Rivière-Environnement, 103 p.

LEMLY A. D., HiLDERBRAND R. H. (2000). «Influence of large woody debris on stream insect communities and benthic detritus ». Hydrobiologia, 421, p. 179-185.

Leveque Ch., MuXart T., AbBadie L., Weil A., VAN Der Leeuw S. (2003). «L'anthroposystème : entité structurelle et fonctionnelle des interactions sociétés-milieux », dans Quelles natures voulons-nous ?, LEVEQUE Ch., VAN DER LEEUW S. (dir.). Paris : Elsevier, p. 110129.

LyAudet P. (1995). Schéma Directeur de Récupération des Déchets Flottants. Le Bouscat: EDF Energie Aquitaine, 45 p.

MARIDET L., COLlin-Huet M.-P. (1994). La végétation aux abords des rivières : sources de vie \& d'équilibre. Paris : Ministère de l'Environnement, $16 \mathrm{p}$.

Maridet L., Piegay H., Gilard O., Thevenet A. (1996). «L'embâcle de bois en rivière : un habitat écologique? un facteur de risques naturels ? ». La Houille Blanche, 5, p. 32-38.

Megahan W. F. (1982). "Channel sediment storage behind obstructions in forested drainage basins draining the granitic bedrock of the Idaho batholic », dans Sediment budgets and routing in forested drainage, Swanson F. J., Janda R. J., Dunne T., Swanston D. N. (dir.). U.S. Forest Service General Technical Report PNW-141, p. 114-121.

Minshall G. W., Minshall J. N. (1977). «Microdistribution of benthic invertebrates in a Rocky mountain (U.S.A.) stream ». Hydrobiologia, 55, p. 231-249.

PIEGAY H. (1996). "Représentation de la biodynamique fluviale : la forêt alluviale de la moyenne Ardèche ». Mappemonde, 3, p. 15-22.

Piegay H., Pautou G., Ruffinoni C. (dir.) (2003). Les forêts riveraines des cours d'eau: écologie, fonctions et gestion. Paris : Institut pour le Développement Forestier, 464 p.

Quintane N. (1999). «Raisons », dans Collectif, L'arbre, Revue Propos de campagne, 9, p. 911.

REICH M., KeRShNER J. L., Wildman R. C. (2003). «Restoring streams with large wood: a synthesis ", dans The Ecology and Management of Wood in World Rivers, GREGORY S. V., BoYer K., Gurnell A. M. (dir.). Bethesda : American Fisheries Society, p. 355-366.

RouX A.L. (1982). Cartographie polythématique appliquée à la gestion écologique des eaux; étude d'un hydrosystème fluvial : le Haut Rhône français. Lyon : rapport CNRS, 116 p.

SCHUMm S.A. (1977). The fluvial system. New York : J. Wiley and Sons, 338 p.

SEDEll J. R., DUVAL W. S. (1985). Influence of forest and rangeland management on anadromous fish habitat in western north America. Portland : USDA Forest Service, General Technical Report PNW-186, 68 p.

TAYlor J., NoDier C., DE CAILleuX A. (1833). Voyages pittoresques et romantiques dans l'ancienne France. Voyages en Languedoc. Paris : Didot, 6 volumes.

THEVENET A. (1998). Intérêt des débris ligneux grossiers pour les poisons dans les grands cours d'eau. Pour une prise en compte de la dimension écologique des débris ligneux grossiers dans la gestion des cours d'eau. Thèse de Doctorat, Lyon I, 100 p.

URABE H., NAKANO S. (1998). «Contribution of woody debris to trout habitat modification in small streams in secondary deciduous forest, northern Japan ». Ecological Research, 13, p. 335345.

Valette P., Gazelle F. (2000). «L'impact des sociétés du XVIII ${ }^{\mathrm{e}}$ et du XIX ${ }^{\mathrm{e}}$ s. sur les paysages garonnais ». Géocarrefour, 75, p. 337-345.

Vannote R. L., Minshall G. W., Cummins K. W., Sedell J. R., Cushing C. E. (1980). « The river continuum concept ». Canadian Journal of Fisheries and Aquatic Sciences, 37, p. 130-137. VERNEILH-PUYRASEAU DE J.-J. (1810). «Observations des commissions consultatives sur le projet de code rural ». Paris : Imprimerie impériale, 2 tomes, 490 et $753 \mathrm{p}$. VILACEQUE J. (2003). «La mémoire de l'eau ». Le Monde, 12 mars 2003. 
Yeats W. B. (1899). Le vent parmi les roseaux. Cognac : Fata Morgana (édition de 1997), 59 p.

\section{Liste des figures}

Figure 1. G. Barnard, «Fontaine Saint-Andéol, Vivarais »(détail), dans Taylor et al., 1835.

Figure 2. L'ancienneté et le vieillissement des ripisylves (533 arbres carottés) occupant les marges de 10 cours d'eau du Sud-Est français (à partir des données de S. Dufour, F. Liébault et H. Piégay). La boîte à moustache permet de présenter synthétiquement l'ensemble des arbres étudiés, avec une valeur centrale, la médiane et la variabilité (indication du premier et du dernier déciles, ainsi que du premier et troisième quartiles). Les cercles correspondent aux individus extrêmes.

Figure 3. Le bois, facteur de diversification physique de la rivière.

Figure 4. Abondance totale et richesse spécifique en fonction du pourcentage de recouvrement (densité de bois). Les limites des classes de recouvrement sont calculées pour obtenir le même nombre d'individus par classe : 1) $0 \%$,2) ]0-5], 3) ]5-15] et 4) >15\%. Les astérisques indiquent une différence significative entre les classes de recouvrement selon le test de Kruskal-Wallis pour $\mathrm{p}<0,01$ (données concernant la Drôme, le Rhône et la Loire, d'après Thévenet, 1998).

Figure 5. S. Hirakawa, 1997. Arbre généalogique/mort. Exposition «Mont-de-Marsan sculpture, Le Japon, Créations in-situ, $1997 »$.

Figure 6. Approche anthroposystémique de la relation hommes/bois en rivière. 
Figure 1. G. Barnard, « Fontaine Saint-Andéol, Vivarais » (détail), dans Taylor et al., 1835.

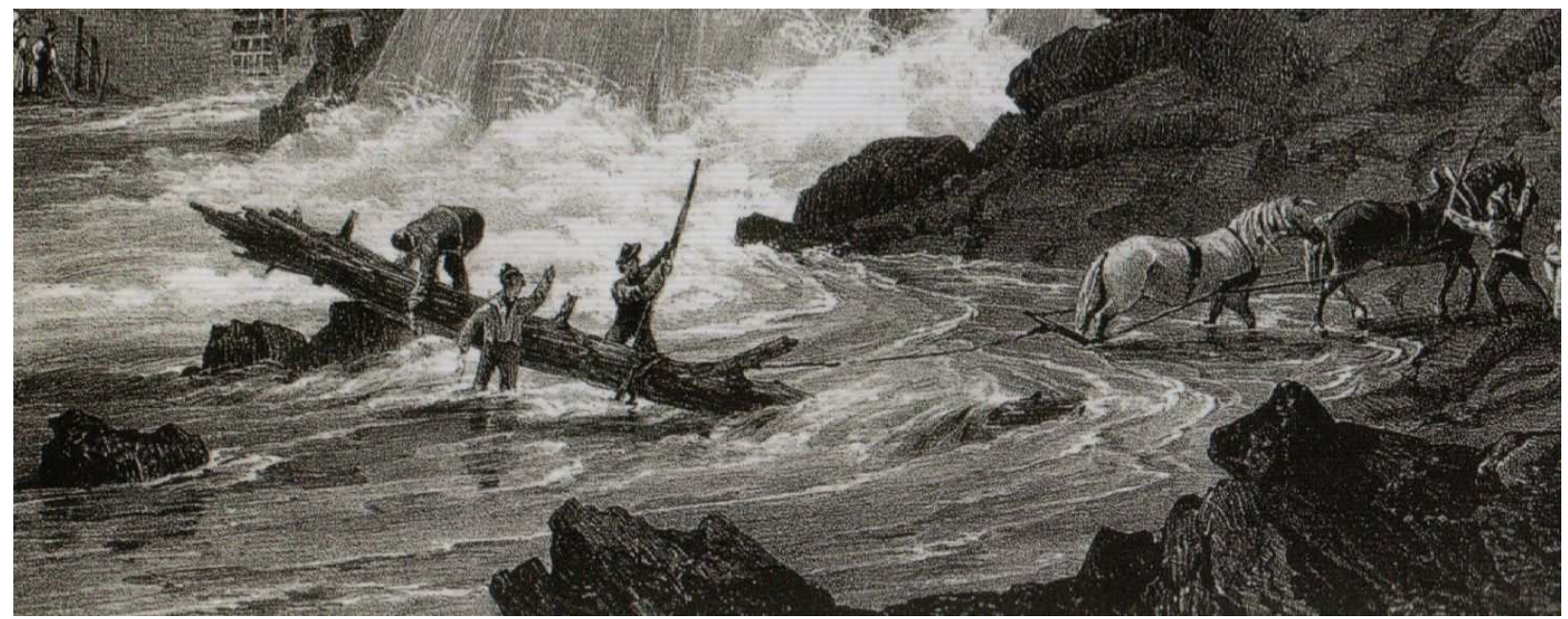


Figure 2. L'ancienneté et le vieillissement des ripisylves (533 arbres carottés) occupant les marges de 10 cours d'eau du Sud-Est français (à partir des données de S. Dufour, F. Liébault et H. Piégay). La boîte à moustache permet de présenter synthétiquement l'ensemble des arbres étudiés, avec une valeur centrale, la médiane et la variabilité (indication du premier et du dernier déciles, ainsi que du premier et troisième quartiles). Les cercles correspondent aux individus extrêmes.

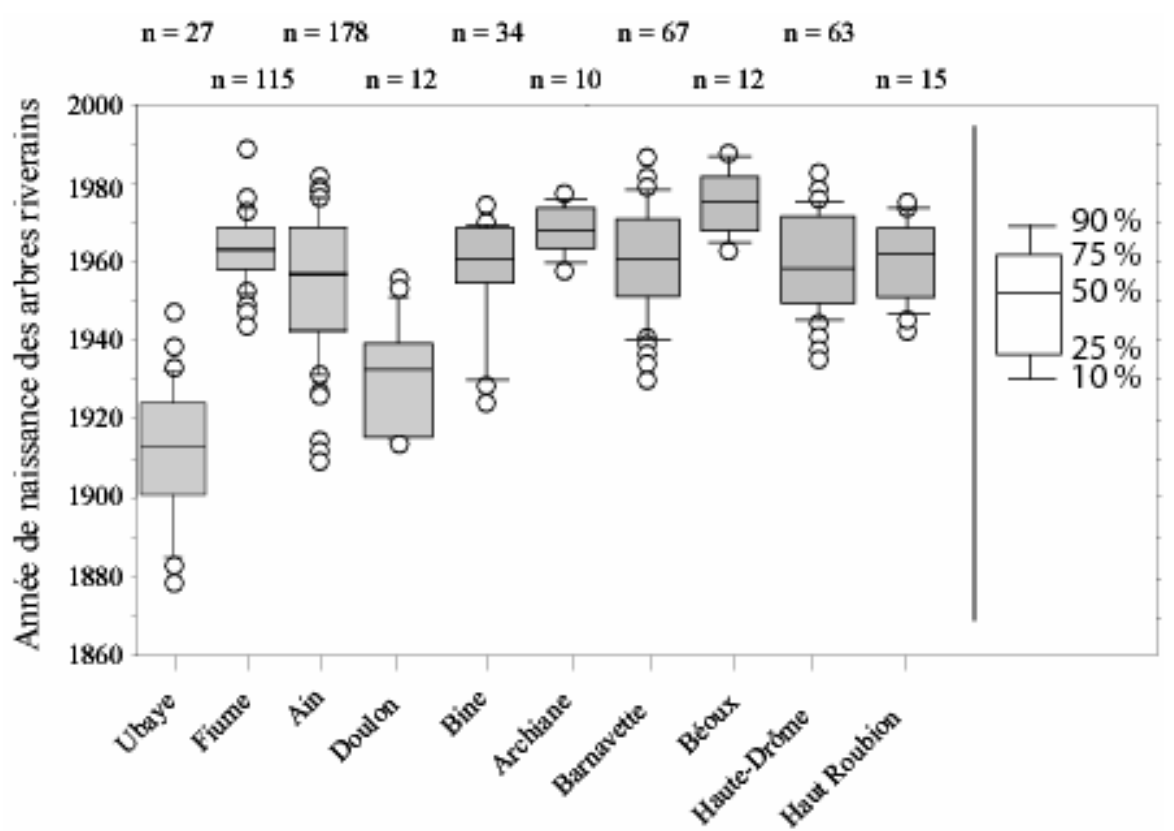


Figure 3. Le bois, facteur de diversification physique de la rivière.

\section{Profondeur et sédimentation}

$\square$ Profond/sable

$\square$ Profond/gravier

Peu profond/caillou

Peu profond/gravier

Peu profond/sable

Peu profond/vase

$\longrightarrow$ Vitesse d'écoulement

Structures de rétention
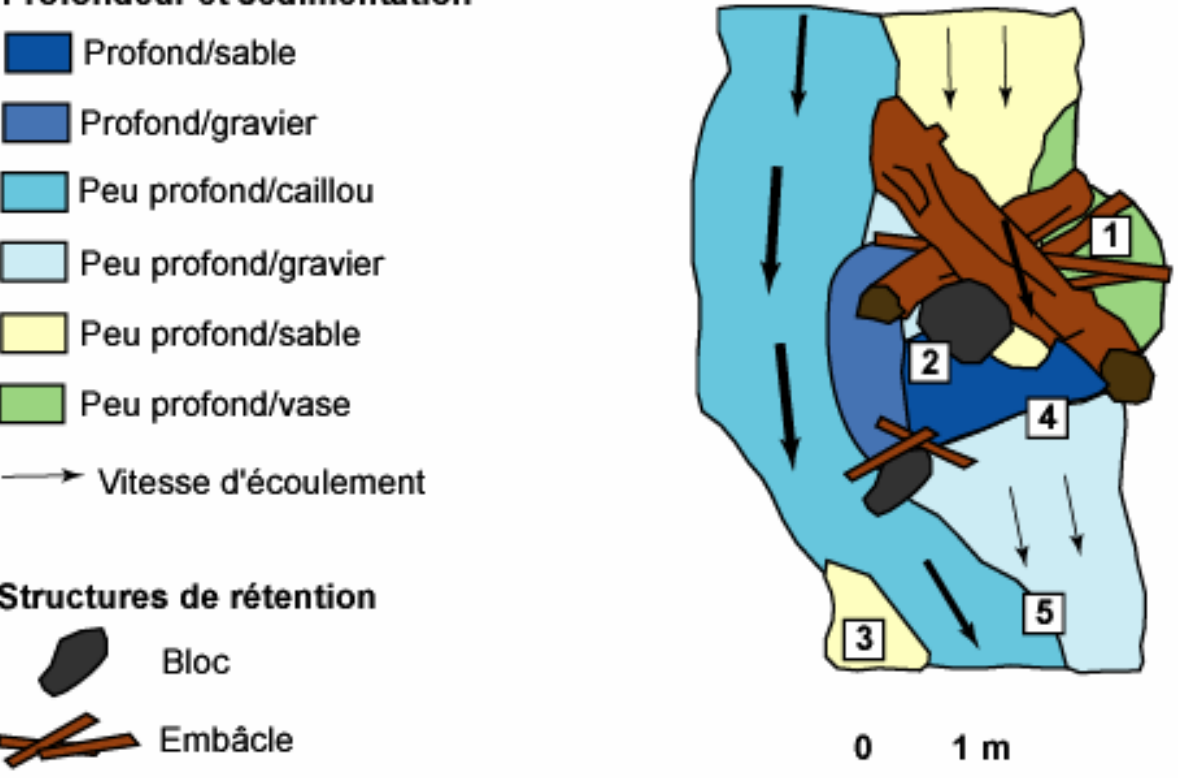

\section{Habitats faunistiques}

1 Zone d'alimentation des invertébrés (accumulation de matière organique)

2 Zone de refuge (caches)
3 Habitat des jeunes stades de poissons

4 Zone d'alimentation des poissons (adultes)

5 Zone de frai 
Figure 4. Abondance totale et richesse spécifique en fonction du pourcentage de recouvrement (densité de bois). Les limites des classes de recouvrement sont calculées pour obtenir le même nombre d'individus par classe : 1) $0 \%$,2) ]0-5], 3) ]5-15] et 4) >15\%. Les astérisques indiquent une différence significative entre les classes de recouvrement selon le test de Kruskal-Wallis pour $\mathrm{p}<0,01$ (données concernant la Drôme, le Rhône et la Loire, d'après Thévenet, 1998).
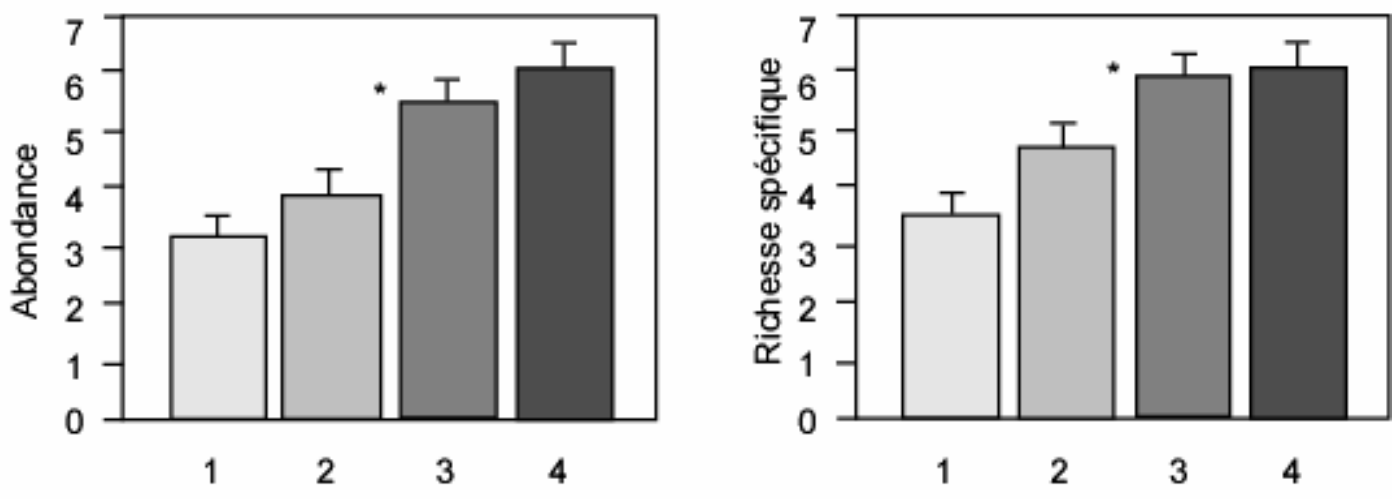
Figure 5. S. Hirakawa, 1997. Arbre généalogique/mort. Exposition « Mont-de-Marsan sculpture, Le Japon, Créations in-situ, 1997 ».

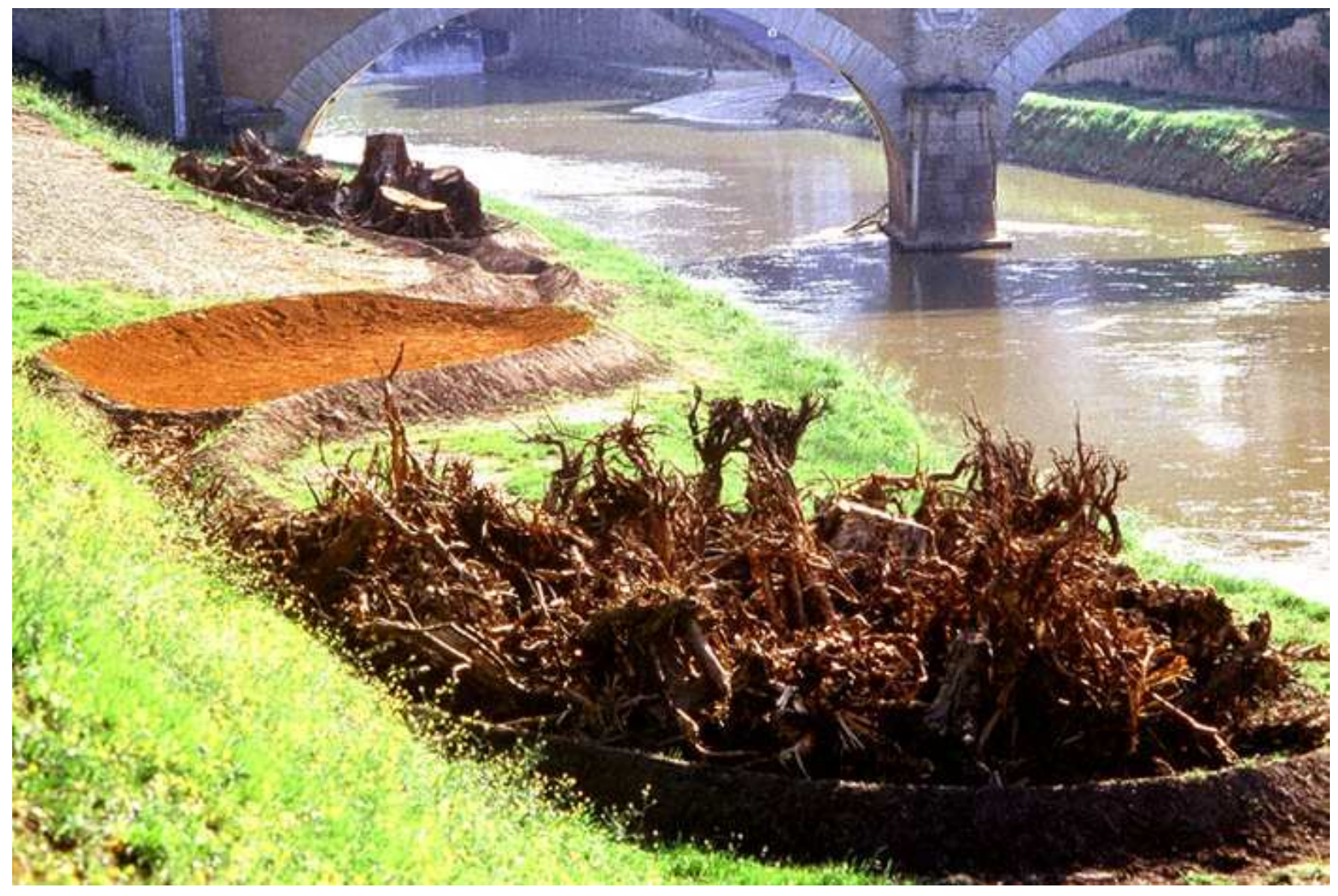


Figure 6. Approche anthroposystémique de la relation hommes/bois en rivière.

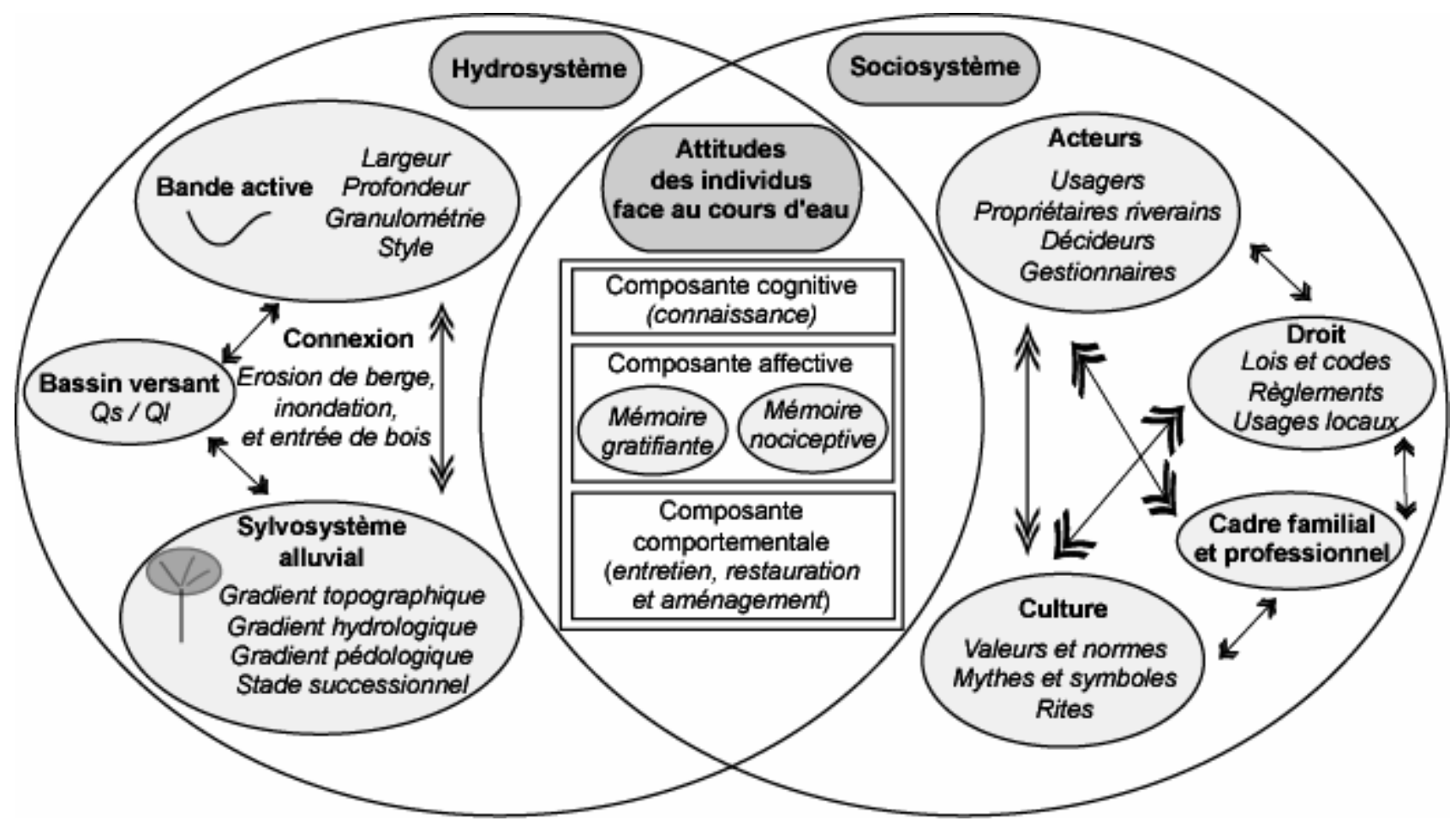

